\title{
A Study on the Difference of Total Grip Strength and Individual Finger Force between Dominant and Non-dominant Hands in Various Grip Spans of Pliers
}

\author{
Yong-Ku Kong ${ }^{1}$, Hyunjoon Park ${ }^{2}$, Dujeong Kim ${ }^{1}$, Taemoon Lee ${ }^{3}$, Eunyoung Roh ${ }^{1}$, \\ Seulki Lee ${ }^{1}$, Wenbin Zhao ${ }^{1}$, Dae-Min Kim ${ }^{1}$, Hyun-Sung Kang ${ }^{1}$ \\ ${ }^{1}$ Department of Industrial Engineering, Sungkyunkwan University, Suwon, 440-746 \\ ${ }^{2}$ Department of Biomechatronics, Sungkyunkwan University, Suwon, 440-746 \\ ${ }^{3}$ Department of Mechanical Engineering, Sungkyunkwan University, Suwon, 440-746
}

\begin{abstract}
Objective: The purpose of this study is to analyze the individual finger force between dominant hand and non-dominant hand and to investigate an effect of the individual finger on the total grip strength depending on dominant hand and non-dominant hand. Background: Many studies on the ratio of the grip force between dominant hand and non-dominant hand has been researched. While a $10 \%$ rule which is a ratio of the grip force between dominant hand and non-dominant hand has been applied in most studies, studies on the rate of the individual finger force between dominant hand and non-dominant hand have been insufficiently researched. Method: The experiment was preceded with 17 subjects (male, mean 25.8 ages). The individual finger force and total grip strength were measured using pliers being able to change the grip span from 45 to $80 \mathrm{~mm}$. Results: The difference of total grip strength between dominant hand and non-dominant hand is following $10 \%$ rule. However, the difference of individual finger force between dominant hand and non-dominant hand are not same as the difference of total grip strength. Especially in the case of grip span with $50 \mathrm{~mm}$, the differences between total grip strength, index finger, middle finger, ring finger, and little finger were $9.87 \pm 14.80 \%, 8.95 \pm 37.17 \%, 13.71 \pm 28.27 \%, 6.77 \pm 24.35 \%$, $39.29 \pm 42.46 \%$, respectively, with $p=0.018$ of statistical significance. Additionally, the results of regression analysis in 50 and $60 \mathrm{~mm}$ of grip span showed that the difference in ring finger affected the most to the total grip strength; and the effects followed in order of index finger, middle finger, and little finger. Conclusion: Our study suggests that an effect of individual finger and grip span of pliers have to be considered when explaining the difference of the total grip strength between dominant hand and non-dominant hand. Application: This result is expected to be used for designing ergonomic hand tool.
\end{abstract}

Keywords: Total grip strength, Individual finger force, Dominant and non-dominant hands

\section{Introduction}

손은 사람이 가장 많이 사용하는 신체부분으로 주로 도구
를 다루는데 사용하기 때문에 일상생활을 정상적으로 유지 하는데 매우 중요한 역할을 하는 신체분절이다. 특히 대부분 의 작업현장 또는 일상생활에서는 과업과 관련된 수공구를 사용하여 작업을 수행하는 것이 보통이므로 이를 보다 효율

Corresponding Author: Hyun-Sung Kang. Department of Industrial Engineering, Sungkyunkwan University, Suwon, 440-746. Phone: +82-31-290-7629, E-mail: kanghs328@naver.com Copyright@2013 by Ergonomics Society of Korea(pISSN:1229-1684 eISSN:2093-8462). All right reserved.

(c) This is an open-access article distributed under the terms of the Creative Commons Attribution Non-Commercial License(http://creativecommons.org/licenses/by-nc/3.0/), which permits unrestricted non-commercial use, distribution, and reproduction in any medium, provided the original work is properly cited. http://www.esk.or.kr 
적이고 안전하게 수행하는 것이 중요한 문제이다. 이를 위해 많은 연구자들은 손의 기능적 측면을 이해하기 위한 연구를 진행하고 있으며, 특히 총 악력(Total grip strength)은 물체 를 쥐고 수공구를 동작시키는데 중요하여 다양한 연구가 진 행되고 있다.

총 악력은 물체를 쥐는 힘으로써, 이를 분석하는 것은 여 러 가지 목적이 있으며 파지폭(Grip span)에 따른 총 악력, 손의 인체측정치(Anthropometry)에 따른 총 악력, 각 손가 락힘(Individual finger force)의 총 악력에 대한 기여도, 주 력손(Dominant hand) 과 비주력손(Non-dominant hand)의 총 악력 차이에 대한 연구 등이 수행되어 왔다. 특히 주력손 과 비주력손의 악력 차이에 대한 연구는 임상적 목적, 표준 데이터 축적, 측정자세와 총 악력 간의 관계 파악, 체력평가 등을 위해 이루어졌다(Kong et al., 2010).

Petersen et al. (1989)은 주력손의 총 악력이 비주력손의 총 악력에 비해 약 $10 \%$ 정도 우위에 있음을 제시하며 $10 \%$ rule을 확인하였으며, Nicolay와 Walker(2005)는 역시 주 력손이 비주력손에 비해 약 $10 \%$ 정도 높은 총 악력을 나 타내지만 더욱 쉽게 피로해지고, 이러한 경향은 남성보다는 여성에서 더 크게 나타난다고 보고하였다. Cetinus et al. (2005)은 당뇨환자들의 총 악력이 정상인 보다 유의하게 적게 나타났으며, 주력손이 비주력손에 비해 약 $4.5 \%$ 높은 총 악력과 파악력을 나타낸다고 하였다. Park et al.(2006) 은 휠체어 테니스 선수들과 의전차 추진 척수손상인의 총 악 력을 분석하여 각 그룹의 주력손과 비주력손에서 차이가 나 타나지 않는다고 하였다. Lee et al. (1995)는 연령대별로 주력손과 비주력손의 총 악력 차이를 조사하였으며, 연령에 따라 그 차이가 달라진다는 결과를 제시하였다. 위의 선행연 구들에서와 같이 총 악력은 대부분의 연구에서 주력손이 비 주력손보다 우세하게 나타나고 있으나 그 차이는 대상의 특 성, 성별, 연령 등에 따라 다소 다른 연구결과를 나타내고 있 으며, Schmidt와 Toews(1970)는 주력손과 비주력손의 총 악력 차이는 개인 별로 다르게 나타나므로 $10 \%$ rule을 적용 할 시 주의해야 한다고 하였다.

총 악력은 파지폭의 변화에도 영향을 받으며 이와 관련된 연구들도 다양하게 보고되었고 대부분의 연구에서는 파지폭 이 최적의 파지폭 범위에서 벗어나면 총 악력이 감소한다 는 연구결과(Jung et al., 2007; Petrofsky et al., 1980; Oh 와 Radwin, 1993; Eksioglu et al., 2004)를 제시하였으며, 특히 작업장에서 주로 사용되는 $\mathrm{A}$ 자형 수공구에 대해서 Fransson 과 Winkel(1991)는 남성의 경우 55 65mm, 여성의 경우 50 60mm의 파지폭에서 최대 악력이 발생된 다고 하였다. 그러나 현재까지의 연구들은 대체로 I자 형태 의 손잡이에 관련된 연구로 편중되어 있으며, 파지폭에 따라 주력손과 비주력손의 각 손가락 힘의 차이를 분석한 연구는
부족한 실정이다.

또한 총 악력은 각 손가락에서 발생하는 힘과 이를 지지하 는 손바닥의 상호작용으로 인해 발생하며, 각 손가락 간의 상호작용에 의해서도 영향을 받는다(Kong et al., 2012). 따 라서 총 악력에 영향을 미치는 각 손가락 별 힘에 대한 연구 도 이루어지고 있으며, Kim et al.(2008)은 MFFM(MultiFinger Force Measurement) System을 사용하여 총 악력 에 대한 각 손가락들의 기여도를 분석하여 중지가 가장 큰 기여도를 나타내고, 소지가 가장 적은 기여도를 나타낸다고 하였다. 이러한 경향은 다른 선행연구들(Kong et al., 2009; Jung et al., 2007; MacDemid et al., 2004)에서도 유사하게 나타나고 있으나, 이 연구들은 주력손의 총 악력에 대한 각 손가락 별 힘의 기여도를 주로 측정하였고, 주력손과 비주력 손에서 나타나는 각 손가락 별 힘의 차이와 그 차이가 총 악 력에 미치는 영향에 대한 연구는 아직까지 미흡한 상태이다.

따라서 본 연구에서는 $\mathrm{A}$ 자 형태의 수공구 중 플라이어 (pliers)에 대해 주력손과 비주력손의 총 악력 및 각 손가 락 별 힘을 비교분석하고, 파지폭에 따라 주력손과 비주력 손의 각 손가락 힘의 차이가 총 악력의 차이에 미치는 영향 을 조사하고자 한다.

\section{Methods}

\subsection{Participants and apparatus}

과거 근골격계질환 병력이 없는 오른손잡이 성인남성 17 명 이 실험에 참여하였으며, 피험자들의 나이는 $25.8 \pm 2.4$ 세이 며, 신장은 $176.1 \pm 3.6 \mathrm{~cm}$, 몸무게는 $70.9 \pm 7.5 \mathrm{~kg}$, 손길이 는 $18.8 \pm 0.9 \mathrm{~cm}$, 손너비는 $8.1 \pm 0.5 \mathrm{~cm}$ 이다.

실험에 사용된 장비는 실제 작업현장에서 사용되는 플라 이어로 헤드부분을 개조하여 파지폭을 조절할 수 있도록 하 였고, 손잡이 부분에는 4 개의 로드셀을 삽입하여 검지부터 소지까지 각 손가락 별 힘을 측정할 수 있도록 자체 제작하 였다. 또한 실험데이터 획득 및 처리를 위해 LabVIEW v8.6 (National Instruments Corporation, Austin, TX, USA)을 사용하였다(Figure 1).

\subsection{Experimental procedures}

파지폭에 따른 총 악력과 각 손가락 별 힘의 변화를 측정 하기 위해 5 개의 파지폭 $(45,50,60,70$ and $80 \mathrm{~mm})$ 과 손가 락(검지, 중지, 약지, 소지)을 독립변수로 설정하였고, 총 악 력은 각 손가락 별 힘의 합으로 산출하였다. 피험자들은 각 파지폭에 대해 주력손과 비주력손을 조합하여 총 10 가지 실 


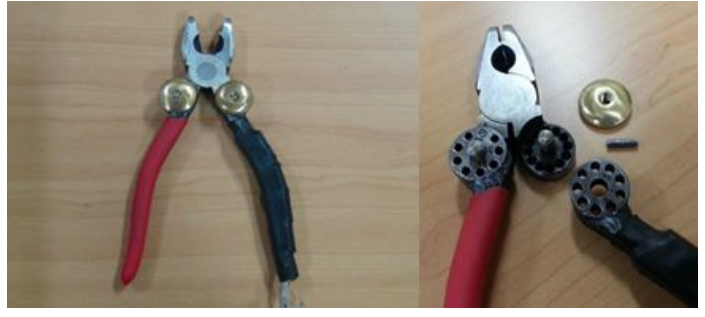

Figure 1. Custom-made plier

험을 수행하였으며 각 실험에 대해 2회씩 반복측정 하였고, 1 회 측정 시 약 5 초 간 악력을 유지하도록 하였다. 각 측정 후에는 약 3 분간의 휴식을 취하도록 하였고, 총 20 번의 측 정은 무작위 순서로 진행하였다. 피험자들에게는 실험 전에 실험 절차와 자세에 대해 충분한 설명을 해주었으며, 플라이 어의 사용 방법에 대해 사전 연습을 수행하도록 하였다. 실 험 자세는 미국 손 치료사 협회 (American Society of Hand Therapist, ASHT) 에서 제안하는 자세와 동일하게 의자에 편히 앉은 상태에서 어깨각도 $0^{\circ}$, 팔꿈치각도 $90^{\circ}$, 손목각도 $0^{\circ}$ 의 자세에서 실험하였다(Figure 2).

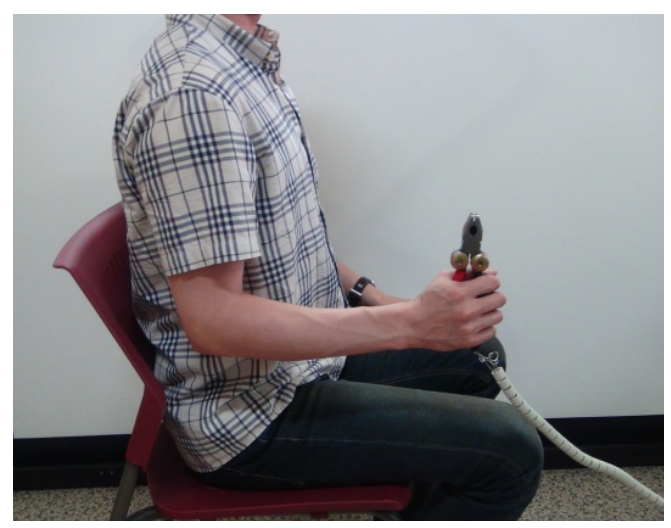

Figure 2. Experiment posture

\subsection{Statistics}

각 피험자에 대한 모든 측정값은 2 회의 반복측정에 대한 평균값을 사용하였다. 주력손과 비주력손의 힘의 차이를 분 석하기 위해 총 악력 및 각 손가락의 구분 여부와 파지폭을 독립변수로 이원분산분석 (Two-way ANOVA)을 수행하 였으며, 각 파지폭에서 총 악력과 각 손가락 별 힘의 차이 를 비교하기 위해 파지폭 별로 일원분산분석(One-way ANOVA)을 수행하였다. 또한 총 악력 및 각 손가락 별 힘 의 차이가 파지폭에 따라 달라지는지 검정하기 위해 파지폭
을 독립변수로 설정하고 총 악력 및 각 손가락 별 힘의 차이 를 종속변수로 설정하여 일원분산분석을 수행하였다. 마지막 으로 각 손가락 별 힘의 차이가 총 악력의 차이에 미치는 영 향을 분석하기 위해 회귀분석을 수행하였으며, 회귀분석 전 회귀모형에 대한 다중공선성 여부를 검토하였다. 모든 통계 분석은 SPSS 통계패키지(SPSS Inc, release 18.0, Chicago, Illinois, USA)를 사용하여 수행하였다.

\section{Results}

\subsection{Comparison of total grip strength and individual finger force differences}

총 악력 및 각 손가락과 파지폭의 변화에 따라 주력손과 비주력손에 대한 힘의 차이를 비교하기 위해 이원분산분석 을 수행한 결과, 파지폭에 대한 주효과 및 두 독립변수 간의 상호작용효과는 통계적으로 유의하지 않았으나 총 악력 및 각 손가락에 대한 주효과는 통계적으로 유의한 차이를 나타 냈다 $(p=0.004)$. 또한 Tukey 검정을 이용한 사후분석 결 과에서는 주력손과 비주력손에 대한 소지의 힘 차이가 총 악력, 중지, 약지와 비교해 각각 통계적으로 유의한 차이(각 각 $p=0.010,0.011,0.016)$ 를 나타냈으며, 다른 손가락들 의 주력손/비주력손에 대한 차이는 총 악력의 차이와 유사하 게 나타났다 $(p>0.05)$.

\subsection{Differences between individual finger force and total grip strength in each grip span}

각 파지폭에서 주력손과 비주력손에 대한 총 악력 및 각 손가락 별 힘의 차이를 일원분산분석을 통해 비교한 결과 파 지폭이 $50 \mathrm{~mm}$ 일 경우 총 악력, 검지, 중지, 약지, 소지의 차 이는 각각 $9.87 \pm 14.80 \%, 8.95 \pm 37.17 \%, 13.71 \pm 28.27 \%$, $6.77 \pm 24.35 \%, 39.29 \pm 42.46 \%$ 로 나타났으며, 통계적으로 유의한 차이를 보였다 $(p=0.018)$. 그러나 다른 파지폭에서 는 통계적으로 유의한 차이가 나타나지 않았다(Table 1).

\subsection{Strength differences between dominant and non- dominant hand accordance to the grip span}

파지폭의 변화에 따른 주력손과 비주력손의 총 악력 및 각 손가락 별 힘의 차이를 일원분산분석을 통해 비교하였다. 먼 저 총 악력의 주력손/비주력손 간 차이는 파지폭이 증가함에 따라 함께 증가하는 추세를 나타내었으나 그 범위가 작고 거 의 $10 \%$ 를 기준으로 약간씩 증가 또는 감소된 값을 나타냈 
다. 또한 파지폭에 따른 총 악력 차이는 통계적으로 유의하 였다 $(p<0.05)$.

검지의 경우 주력손과 비주력손의 차이가 8.86 23.40\% 로 나타났으며, 파지폭이 작을 때 가장 작은 차이가 나타났 고, 파지폭이 $70 \mathrm{~mm}$ 이상일 때 $22 \%$ 이상의 차이가 나타났 다. 중지의 경우에는 힘의 차이가 8.31 13.71\%로 나타났 으며, 약지와 소지의 힘 차이는 각각 5.94 15.83\%, 20.33 $39.29 \%$ 의 범위를 나타냈다. 그러나 모든 손가락에서 주력 손과 비주력손의 힘 차이는 파지폭에 따라 통계적으로 유의 한 차이를 나타내지 않았다(Table 1).

Table 1. Mean and S.D. of Strength differences between dominant and non -dominant, when grip span and individual finger force were considered

(unit: \%)

\begin{tabular}{c|c|c|c|c|c|c}
\hline \multirow{2}{*}{$\begin{array}{c}\text { Grip } \\
\text { span }\end{array}$} & \multicolumn{2}{|c|}{ Difference of dominant and non-dominant hand } & \multirow{2}{*}{$\mathrm{P}_{\mathrm{f}}$} \\
\cline { 2 - 6 } & Total & Index & Middle & Ring & Little & \\
\hline $\begin{array}{c}45 \\
(\mathrm{~mm})\end{array}$ & $\begin{array}{c}8.42 \\
(21.98)\end{array}$ & $\begin{array}{c}8.86 \\
(28.95)\end{array}$ & $\begin{array}{c}8.31 \\
(27.22)\end{array}$ & $\begin{array}{c}15.83 \\
(40.15)\end{array}$ & $\begin{array}{c}22.66 \\
(74.15)\end{array}$ & 0.827 \\
\hline $\begin{array}{c}50 \\
(\mathrm{~mm})\end{array}$ & $\begin{array}{c}9.87^{\mathrm{AB}} \\
(14.18)\end{array}$ & $\begin{array}{c}8.95^{\mathrm{A}} \\
(37.17)\end{array}$ & $\begin{array}{c}13.71^{\mathrm{AB}} \\
(28.27)\end{array}$ & $\begin{array}{c}6.77^{\mathrm{A}} \\
(24.35)\end{array}$ & $\begin{array}{c}39.29^{\mathrm{B}} \\
(42.46)\end{array}$ & $\mathbf{0 . 0 1 8}$ \\
\hline $\begin{array}{c}60 \\
(\mathrm{~mm})\end{array}$ & $\begin{array}{c}9.22 \\
(12.55)\end{array}$ & $\begin{array}{c}6.94 \\
(29.96)\end{array}$ & $\begin{array}{c}8.89 \\
(19.54)\end{array}$ & $\begin{array}{c}10.74 \\
(23.31)\end{array}$ & $\begin{array}{c}26.89 \\
(37.21)\end{array}$ & 0.165 \\
\hline $\begin{array}{c}70 \\
(\mathrm{~mm})\end{array}$ & $\begin{array}{c}10.78 \\
(13.34)\end{array}$ & $\begin{array}{c}23.40 \\
(43.34)\end{array}$ & $\begin{array}{c}10.56 \\
(16.40)\end{array}$ & $\begin{array}{c}5.94 \\
(18.73)\end{array}$ & $\begin{array}{c}20.33 \\
(29.41)\end{array}$ & 0.280 \\
\hline $\begin{array}{c}80 \\
(\mathrm{~mm})\end{array}$ & $\begin{array}{c}11.66 \\
(12.81)\end{array}$ & $\begin{array}{c}22.35 \\
(51.14)\end{array}$ & $\begin{array}{c}9.19 \\
(15.56)\end{array}$ & $\begin{array}{c}13.76 \\
(17.01)\end{array}$ & $\begin{array}{c}21.35 \\
(41.34)\end{array}$ & 0.674 \\
\hline $\mathrm{P}_{\mathrm{s}}$ & 0.977 & 0.577 & 0.956 & 0.763 & 0.767 & - \\
\hline
\end{tabular}

*Total: Total grip strength, Index: Index finger force, Middle: Middle finger force, Ring: Ring finger force, Little: Little finger force.

*A, B: Divided groups based on Tukey test.

$* P_{\text {f: }} P$-value for ANOVA result in each grip span, when total grip strength and individual finger force are considered as independent variables.

${ }^{*} \mathrm{P}_{\mathrm{s}}: P$-value for ANOVA result in individual fingers, when each grip span is considered as independent variables.

\subsection{The effects of individual finger force differences on total grip strength differences}

회귀분석을 통하여 주력손과 비주력손의 총 악력 차이 에 영향을 미치는 각 손가락 별 영향력을 분석하였다. 먼저 회귀모형은 통계적으로 유의한 모형이 구성되었으며 $(p=$ $0.000)$, 각 독립변수 간의 다중공선성을 검토하기 위해 각 손가락의 분산팽창계수(VIF), Durbin-Watson(DW) 통계 량을 산출한 결과 각각 $1 \sim 2.5,10$ 이하의 값을 나타냈다.
이는 각 독립변수간의 자기상관이 매우 적다는 것을 나타내 므로 각 변수들은 독립성을 가진다고 할 수 있다.

Table 2는 각 파지폭에서 각 손가락 별 힘의 차이가 총 악력 차이에 상대적으로 미치는 영향을 회귀분석의 표준화 계수를 통해 분석한 결과이다. 파지폭이 $45 \mathrm{~mm}$ 일 때에는 중 지에서 0.480으로 가장 영향력이 컸으며 파지폭 $50,60 \mathrm{~mm}$ 에서는 각각 $0.622,0.624$ 로 약지가 가장 큰 영향을 미치 는 것으로 나타났다. 파지폭 $70,80 \mathrm{~mm}$ 에서는 검지에서 각 각 $0.477,0.511$ 의 값으로 가장 큰 영향을 미치는 것으로 나타났다. 또한 일반적으로 가장 큰 총 악력을 발휘하는 50 , $60 \mathrm{~mm}$ 의 파지폭에서 주력손과 비주력손 간의 총 악력 차이 에 영향을 미치는 손가락의 순위는 약지, 검지, 중지, 소지 순으로 나타났으며, 각 파지폭에 따라 이 순위가 다르게 나 타났다.

Table 2. The effects of individual finger force differences on total grip strength difference(Standardized coefficients in regres sion analysis)

\begin{tabular}{c|c|c|c|c}
\hline Grip span $(\mathrm{mm})$ & Index & Middle & Ring & Little \\
\hline 45 & 0.388 & $\mathbf{0 . 4 8 0}$ & 0.315 & 0.144 \\
\hline 50 & 0.596 & 0.445 & $\mathbf{0 . 6 2 2}$ & 0.223 \\
\hline 60 & 0.466 & 0.357 & $\mathbf{0 . 6 2 4}$ & 0.278 \\
\hline 70 & $\mathbf{0 . 4 7 7}$ & 0.475 & 0.371 & 0.324 \\
\hline 80 & $\mathbf{0 . 5 1 1}$ & 0.357 & 0.403 & 0.510 \\
\hline
\end{tabular}

\section{Discussion}

주력손과 비주력손의 총 악력 차이는 파지폭에 따라 달 라지지만, 일반적으로 $10 \%$ rule이라고 알려져 있는 연구 (Petersen et al., 1989) 결과와 유사하게 약 8.31 11.66\% 의 차이를 보였다. 이는 파지폭이 변하더라도 주력손과 비주 력손의 총 악력 차이는 비슷한 수준을 유지한다는 것을 의미 한다. 그러나 중지를 제외한 다른 손가락들에서는 파지폭이 변함에 따라 주력손과 비주력손의 힘 차이가 총 악력에 비 해 큰 폭으로 변하였으며, 특히 소지와 검지에서는 그 범위 가 각각 20.33 39.29\%, 6.94 23.40\%로 다른 손가락들에 비해 상대적으로 크게 나타났다. 이와 같은 결과는 각 손가 락들에서는 파지폭이 변함에 따라 주력손과 비주력손의 차 이가 다르게 나타난다는 것을 의미하며 Tukey 검정의 결과 에서 소지의 차이가 다른 손가락 및 총 악력의 차이와 통계 적으로 유의하게 차이가 난다는 결과는 이를 뒷받침하는 것 이라 할 수 있다. 
또한 같은 파지폭에서도 주력손과 비주력손의 힘 차이가 각 손가락 별로 다르게 나타났는데 특히 파지폭이 $50 \mathrm{~mm}$ 인 경우에는 소지의 차이가 다른 손가락들의 차이에 비해 통계 적으로 유의하게 다른 것으로 나타났으며, 총 악력의 차이와 도 다르게 나타났다. 이는 총 악력의 차이가 약 $10 \%$ 정도로 나타나더라도 각 손가락 별 힘의 차이는 모든 손가락에서 $10 \%$ 로 나타나지는 않는다는 것을 의미한다. 따라서, 각 손 가락 별 힘의 차이가 총 악력의 차이에 미치는 영향이 다를 것이라 생각된다.

회귀분석의 표준화계수를 통해 주력손과 비주력손 간의 총 악력 차이를 발생시키는 각 손가락의 영향을 분석한 결과 같은 파지폭에서도 손가락에 따라 다른 정도의 영향을 미치 는 것으로 나타났으며, 파지폭이 변함에 따라 같은 손가락의 영향력도 달라지고, 각 손가락들의 영향력 순위도 달라지는 것으로 나타났다. MacDemid et al. (2004)은 총 악력에 대한 각 손가락 별 힘의 기여도가 왼손과 오른손에 차이가 있다고 하였으며, $\operatorname{Seo}(2012)$ 는 파지폭에 따라 총 악력에 대한 손 가락 별 기여도가 다르다고 하였는데, 위와 같은 선행연구의 결과를 고려하였을 때 파지폭에 따라 주력손과 비주력손의 총 악력 차이에 영향을 미치는 손가락이 달라질 수 있다고 판단된다.

또한 일반적으로 최대 악력이 도출되는 $50,60 \mathrm{~mm}$ 의 파지 폭에서 주력손과 비주력손 간의 총 악력 차이를 발생시키는 손가락은 약지, 검지, 중지, 소지 순으로 동일한 결과를 도출 하였다. 이에 따라 최대 악력이 도출될 때 주력손과 비주력 손 간의 총 악력 차이를 발생시키는 손가락은 약지, 검지, 중 지, 소지 순으로 생각할 수 있다.

\section{Conclusions}

본 연구는 A 타입 손잡이 형태를 가지는 수공구를 사용하 여 파지폭에 따른 주력손과 비주력손 간 총 악력 차이와 총 악력 차이에 영향을 주는 손가락에 대해 분석하여 다음과 같 은 결론을 도출하였다.

1. 파지폭이 달라지더라도 주력손과 비주력손 간의 총 악 력 차이는 기존에 알려진 $10 \%$ rule과 유사하다.

2. 주력손과 비주력손 간의 총 악력의 차이가 약 $10 \%$ 정 도라도 각 손가락에서 나타나는 차이는 이와 다르며, 총 악력 차이에 대한 각 손가락의 영향력이 다르다.

3. 파지폭에 따라 총 악력의 차이에 영향을 주는 손가락 의 우선순위가 다르다.
본 연구에서는 A형 수공구 중 플라이어에 한하여 결과를 도출하였으며, 총 악력의 차이에 대한 각 손가락 별 힘 차이 의 기여도를 산출하지 못한 제한점이 있다. 따라서, 향후 연 구에서는 다양한 형태의 수공구와 악력에 영향을 미치는 성 별, 연령, 직업 등의 사항을 고려하여 주력손과 비주력손의 힘 차이와 각 손가락 별 기여도를 분석해야 할 것으로 생각 되며, 이러한 연구결과는 수공구 설계와 손 치료를 위한 임 상분야에서 활용될 수 있을 것으로 기대된다.

\section{Acknowledgements}

This work was supported by the National Research Foundation of Korea(NRF) grant funded by the Korean government(MEST) (No. 2011-0016753).

\section{References}

Cetinus, E., Buyukbese, M., Uzel, M., Ekerbicer, H. and Karaoguz, A., Hand grip strength in patients with Type 2 diabetes mellitus, Diabetes Research and Clinical Practice, 70, 278-286, 2005.

Eksioglu, M., Relative optimum grip span as a function of hand anthropometry, International Journal of Industrial Ergonomics, 34, 1-12, 2004.

Fransson, C. and Winkel, J., Hand Strength: the influence of grip span type, Ergonomics, 34, 881-892, 1991.

Jung, M.C., Kim, D.M. and Kong, Y.K., Evaluation of Individual Finger Force to Grip Strength in Various Grip Spans and Hand Sizes Journal of the Ergonomics Society of Korea, 26(3), 59-65, 2007.

Kim, D.M. and Kong, Y.K., Research of Grip Forces and Subjective Preferences for Various Individual Finger Grip Spans by using an "Adjustable Multi-Finger Force Measurement(MFFM) System", Applied Human Factors and Ergonomics, 2nd International Conference, July 14-17, Las Vegas, USA, 2008.

Kong, Y.K., Sohn, S.T. and Han, J.G., A Review Study for Grip Strengths of Hand, Journal of the Ergonomics Society of Korea, 29(5), 715-725, 2010.

Kong, Y.K., Seo, M.T., Kang, H.S. and Kim, D.M., Effect of finger interaction on individual finger: index finger, Work: A Journal of Prevention, Assessment and Rehabilitation, 41, 5696-5698, 2012.

Kong, Y.K., Sohn, S.T., Kim, D.M. and Jung, M.C., Grip Force, Finger Force, and Comfort analyses of Young and Old People by Hand Tool Handle Shapes, Journal of the Ergonomics Society of Korea, 28(2), 27-34, 2009.

Lee, K.S., Woo, K.J., Shim, J.H. and Lee, G.H., The Clinical Study of Grip and Pinch Strength in Normal Korean Adult, Journal of Korean Prthop. 
Assoc., 30(6), 1589-1597, 1995.

MacDemid, J.C., Lee, A., Richards, R.S. and Roth, J.H., Individual finger strength: Are the ulnar digits "powerful"?, Journal of Hand Therapy, 17(3), 363-367, 2004.

Nicolay, C. and Walker, A., Grip strength and endurance: influences of anthropometric variation, hand dominance, and gender, International Journal of Industrial Ergonomics, 35, 605-618, 2005.

Oh, S. and Radwin, R.G., Pistol grip power tool handle and trigger size effects on grip exertions and operator preference, Human Factors, 35(3), 551-569, 1993.

Park, S., Jung, S.H. and Kim, T.Y., Effect of Wheelchair Tennis Exercise on Hand Strength of Wheelchair Propelling Spinal Cord Injured Patients, The Korea Jurnal of Sports Science, 15(4), 859-868, 2006.

Petersen, P., Petrich, M., Connor, H. and Conklin, D., Grip strength and hand dominance: challenging the $10 \%$ rule, The American Journal of Occupational Therapy, 43(7), 444-447, 1989.

Petrofsky, J.S., Williams, C., Kamen, G. and Lind, A.R., The effect of handgrip span on isometric exercise performance, Ergonomics, 23(12), 1129-1135, 1980.

Schmidt, R.T. and Toews, J.V., Grip strength as measured by the Jamar dynamometer, Archives of Physical Medicine and Rehabilitation, 51(6), 321-327, 1970.

Seo, M.T., (A) study on determining the optimal grip span of pliers in A-type hand-tool, Master's Thesis, Department of Industrial Engineering, Sungkyunkwan University, 50, 2012.

\section{Author listings}

Yong-Ku Kong: ykong@skku.edu

Highest degree: $\mathrm{PhD}$, Industry Engineering, the Pennsylvania State University

Position title: Professor, Department of Industrial Engineering, Sungkyunkwan University

Areas of interest: Ergonomic Product Design, Musculoskeletal Disorders

\section{Hyunjoon Park: hyunjun21@skku.edu}

Highest degree: B.S., Department of Biomechatronics Engineering, Sungkyunkwan University

Position title: Ph.D. Candidate, Department of Biomechatronics Engineering, Sungkyunkwan University

Areas of interest: Musculoskeletal disorder, Digital human modeling, Motion analysis, Ergonomic simulation
Dujeong Kim: aspiria@skku.edu

Highest degree: B.S., Department of Systems Management Engineering, Sungkyunkwan University

Position title: M.S, Department of Industrial Engineering, the University of Sungkyunkwan

Areas of interest: Human-Computer Interaction, Accessibility, User Interface, User Experience, Universal Design

Taemoon Lee: tmlee@skku.edu

Highest degree: Master of Mechanical Engineering, Department of Mechanical Entineering, Sungkyunkwan University

Position title: Ph.D Candidate, Department of Mechanical Engineering, Sungkyunkwan University

Areas of interest: Feature Recognition, Feature Extraction, Computer Aided Design(CAD), Computer Aided Process Planning(CAPP), Manufacturing Execution System, Business Process Management, Knowledge Management

Eunyoung Roh: eynoh88@gmail.com

Highest degree: B.S., Department of Systems Management Engineering, Sungkyunkwan University

Position title: M.S. Candidate, Department of Industrial Engineering, Sungkyunkwan University

Areas of interest: Human-Computer Interaction, Accessibility, User Interface, User Experience, Universal Design

Sulki Lee: sgsaid@skku.edu

Highest degree: B.S., Computer and Communication Engineering, Kangwon National University

Position title: M.S, Department of Industrial Engineering, the University of Sungkyunkwan

Areas of interest: Human-Computer Interaction, Accessibility, User Interface, User Experience, Universal Design

Wenbin Zhao: wenbin86@skku.edu

Highest degree: Master of Science in Engineering (Industrial Engineering), Department of Industrial Engineering, Sungkyunkwan University

Position title: Ph.D. Candidate, Department of Industrial Engineering, Sungkyunkwan University

Areas of interest: Digital Virtual Manufacturing/PLM, Concurrent \& Collaborative Engineering, e-Manufacturing, Sustainable Manufacturing, Manufacturing System and Information

\section{Dae-Min Kim: kimdaemin@skku.edu}

Highest degree: M.S., Department of Systems Management Engineering, Sungkyunkwan University

Position title: Ph.D. Candidate, Department of Industrial Engineering, Sungkyunkwan University

Areas of interest: Ergonomic Product Design, Musculoskeletal Disorders 
Hyun-Sung Kang: kanghs328@gmail.com

Highest degree: B.S., Department of Systems Management Engineering,

Sungkyunkwan University

Position title: M.S. Candidate, Department of Industrial Engineering,

Sungkyunkwan University

Areas of interest: Musculoskeletal Simulation, Motion Capture Analysis,

Bioelectric Signal Analysis, Ergonomic Product Design, Hand tools
Date Received : 2013-11-15

Date Revised :2013-12-05

Date Accepted : 2013-12-10 